\title{
On Spectrum of Differential Operator with Block-Triangular Matrix Coefficients
}

\author{
A.M. Kholkin \\ Pryazovskyi State Technical University \\ 7 Universitets'ka Str., Mariupol 87500, Ukraine \\ E-mail: a.kholkin@gmail.com \\ F.S. Rofe-Beketov \\ B. Verkin Institute for Low Temperature Physics and Engineering \\ National Academy of Sciences of Ukraine \\ ${ }_{47}$ Lenin Ave., Kharkiv, 61103, Ukraine \\ E-mail: rofebeketov@ilt.kharkov.ua
}

Received November 5, 2012, revised July 15, 2013

For the Sturm-Louville equation with block-triangular matrix potential that increases at infinity, both increasing and decreasing at infinity matrix solutions are found. The structure of spectrum for the differential operator with these coefficients is defined.

Key words: differential operator, spectrum, block-triangular matrix coefficients.

Mathematics Subject Classification 2010: 34K11, 47A10.

Dedicated to our Teacher, Academician Vladimir A. Marchenko on the occasion of his 90th birthday, with appreciation and admiration for his contribution to spectral theory, differential equations and mathematical physics

The study of the relationship between spectral and oscillation properties of non-selfadjoint differential operators with block-triangular matrix coefficients that increase at infinity [1] includes the study of the structure of the spectra of these operators. For the case of an operator with decreasing at infinity triangular matrix potential and a bounded first moment, the structure of the spectrum in the context of the inverse scattering problem was established in [2-4].

In $[5,6]$, V.A. Marchenko introduced a notion of the generalized spectral function $R$ for a Sturm-Liouville operator with arbitrary complex valued potential on the semiaxis. This result was generalized to the case of non-selfadjoint systems $[7,8]$. The distribution (the matrix one in the case of systems) acts on

(C) A.M. Kholkin and F.S. Rofe-Beketov, 2014 
the topological space of test functions. It determines the formulas of expansion in eigenfunctions and also allows one to solve the inverse problem of spectral analysis for the non-selfadjoint case. In the case of selfadjoint problems, the distribution is generated by a non-negative measure (either a scalar or a matricial one in the case of systems). We are interested in analyzing a specific form of a spectrum and spectral matricial distribution $R$ for some classes of non-selfadjoint systems. In [3], while solving the inverse scattering problem on the semiaxis for the case of triangular matrix potentials, the Parseval equality was established, and thus a form of spectral matrix distribution of V.A. Marchenko type was found (for the selfadjoint case, see [9]). We obtain conditions which guarantee the discreteness of spectrum for a broad class of Sturm-Liouville operators on the semiaxis with block-triangular matrix potentials, whose diagonal blocks are Hermitian matrices. For these potentials, a form of V.A. Marchenko type spectral matricial distribution is found. Note that this distribution, under the presence of multiple poles for the resolvent, does not reduce to a matrix measure even in the non-selfadjoint case. These results are applied to extending the Sturm type oscillation theory from selfadjoint systems [10] to systems with triangular matrix potentials [1].

Consider the equation with a block-triangular matrix potential

$$
l[\bar{y}]=-\bar{y}^{\prime \prime}+V(x) \bar{y}=\lambda \bar{y}, \quad 0 \leq x<\infty,
$$

where

$$
V(x)=v(x) I_{m}+U(x), \quad U(x)=\left(\begin{array}{cccc}
U_{11}(x) & U_{12}(x) & \ldots & U_{1 r}(x) \\
0 & U_{22}(x) & \ldots & U_{2 r}(x) \\
\ldots & \ldots & \ldots & \ldots \\
0 & 0 & \ldots & U_{r r}(x)
\end{array}\right)
$$

$v(x)$ is a real scalar function such that $0<v(x) \rightarrow \infty$ monotonically, as $x \rightarrow \infty$, with monotonic absolutely continuous derivative. Also, $U(x)$ is a relatively small perturbation, e.g., $|U(x)| v^{-1}(x) \rightarrow 0$ as $x \rightarrow \infty$ or $|U| v^{-1} \in L^{\infty}(\mathbb{R})$. The diagonal blocks $U_{k k}(x), k=\overline{1, r}$, are assumed to be $m_{k} \times m_{k}$ Hermitian matrices with $m_{k} \geq 1$ (in particular, with $m_{k}=1$ these blocks are just real scalar functions).

Let $\sum_{k=1}^{r} m_{k}=m$, and denote by $I_{m}$ the unit $m \times m$ matrix.

Denote by $H_{m}$ the $m$-dimensional Hilbert space. A vector $\bar{h} \in H_{m}$ will be written in the form $\bar{h}=\operatorname{col}\left(\bar{h}_{1}, \bar{h}_{2}, \ldots, \bar{h}_{r}\right)$, where $\bar{h}_{k}, k=\overline{1, r}$, is a vector from $H_{m_{k}}$. Thus we have $\bar{y}=\operatorname{col}\left(\bar{y}_{1}, \bar{y}_{2}, \ldots, \bar{y}_{r}\right)$, where $\bar{y}_{k} \in H_{m_{k}}$.

I. Let us start with considering the case when

$$
v(x) \geq C x^{2 \alpha}, \quad C>0, \quad \alpha>1 .
$$


(The spectral properties of one-dimensional Schrödinger operator with polynomial potential is the subject of [11]).

Assume that the coefficients of (1) satisfy the conditions

$$
\begin{gathered}
\int_{0}^{\infty}|U(t)| v^{-\frac{1}{2}}(t) d t<\infty, \\
\int_{0}^{\infty} v^{\prime 2}(t) v^{-\frac{5}{2}}(t) d t<\infty, \quad \int_{0}^{\infty} v^{\prime \prime}(t) v^{-\frac{3}{2}}(t) d t<\infty .
\end{gathered}
$$

Rewrite equation (1) in the form

$$
-\bar{y}^{\prime \prime}+(v(x)+q(x)) \bar{y}=\left((\lambda+q(x)) I_{m}-U(x)\right) \bar{y},
$$

where $q(x)$ is given by (cf. $[12,13])$

$$
q(x)=\frac{5}{16}\left(\frac{v^{\prime}(x)}{v(x)}\right)^{2}-\frac{1}{4} \frac{v^{\prime \prime}(x)}{v(x)} .
$$

Consider the functions

$$
\begin{aligned}
& \gamma_{0}(x, \lambda)=\frac{1}{\sqrt[4]{4 v(x)}} \exp \left(-\int_{0}^{x} \sqrt{v(u)} d u\right), \\
& \gamma_{\infty}(x, \lambda)=\frac{1}{\sqrt[4]{4 v(x)}} \exp \left(\int_{0}^{x} \sqrt{v(u)} d u\right) .
\end{aligned}
$$

It is easy to see that with $x \rightarrow \infty$, one has

$$
\gamma_{0}(x, \lambda) \rightarrow 0, \quad \gamma_{\infty}(x, \lambda) \rightarrow \infty .
$$

These solutions constitute a fundamental system of solutions of the scalar differential equation

$$
-z^{\prime \prime}+(v(x)+q(x)) z=0
$$

in such a way that for all $x \in[0, \infty)$ one has

$$
W\left(\gamma_{0}, \gamma_{\infty}\right):=\gamma_{0}(x, \lambda) \gamma_{\infty}^{\prime}(x, \lambda)-\gamma_{0}^{\prime}(x, \lambda) \gamma_{\infty}(x, \lambda)=1
$$


Theorem 1. Under conditions (3), (4), (5), equation (1) has a unique decreasing at infinity $m \times m$ matrix solution $\Phi(x, \lambda)$, satisfying the conditions

$$
\lim _{x \rightarrow \infty} \frac{\Phi(x, \lambda)}{\gamma_{0}(x, \lambda)}=I_{m}
$$

and

$$
\lim _{x \rightarrow \infty} \frac{\Phi^{\prime}(x, \lambda)}{\gamma_{0}^{\prime}(x, \lambda)}=I_{m}
$$

Also, there exists a unique increasing at infinity $m \times m$ matrix solution $\Psi(x, \lambda)$, satisfying the conditions

$$
\lim _{x \rightarrow \infty} \frac{\Psi(x, \lambda)}{\gamma_{\infty}(x, \lambda)}=I_{m}
$$

and

$$
\lim _{x \rightarrow \infty} \frac{\Psi^{\prime}(x, \lambda)}{\gamma_{\infty}^{\prime}(x, \lambda)}=I_{m}
$$

P r o o f. 1. Equation (6) allows one to derive the integral equation

$$
\Phi(x, \lambda)=\gamma_{0}(x, \lambda) I_{m}+\int_{x}^{\infty} K(x, t, \lambda) \Phi(t, \lambda) d t
$$

where

$$
\begin{gathered}
K(x, t, \lambda)=C(x, t)\left[(\lambda+q(t)) I_{m}-U(t)\right], \\
C(x, t)=\gamma_{\infty}(x, \lambda) \gamma_{0}(t, \lambda)-\gamma_{\infty}(t, \lambda) \gamma_{0}(x, \lambda),
\end{gathered}
$$

with $C(x, t)$ being the Cauchy function that in each variable satisfies equation (10) and the initial conditions

$$
\left.C(x, t)\right|_{x=t}=0,\left.\quad C_{x}^{\prime}(x, t)\right|_{x=t}=1,\left.\quad C_{t}^{\prime}(x, t)\right|_{x=t}=-1 .
$$

Set

$$
\chi(x, \lambda)=\frac{\Phi(x, \lambda)}{\gamma_{0}(x, \lambda)}
$$

to rewrite equation (15) in the form

$$
\chi(x, \lambda)=I_{m}+\int_{x}^{\infty} R(x, t, \lambda) \chi(t, \lambda) d t,
$$

where

$$
R(x, t, \lambda)=K(x, t, \lambda) \frac{\gamma_{0}(t, \lambda)}{\gamma_{0}(x, \lambda)}
$$


In view of

$$
\begin{array}{r}
\left|C(x, t) \frac{\gamma_{0}(t, \lambda)}{\gamma_{0}(x, \lambda)}\right|=\left|\gamma_{0}^{2}(t) \frac{\gamma_{\infty}(x, \lambda)}{\gamma_{0}(x, \lambda)}-\gamma_{0}(t, \lambda) \gamma_{\infty}(t, \lambda)\right| \\
=\left|\frac{1}{2 \sqrt{v(t)}} \exp \left(-2 \int_{0}^{t} \sqrt{v(u)} d u\right) \exp \left(2 \int_{0}^{x} \sqrt{v(u)} d u\right)-\frac{1}{2 \sqrt{v(t)}}\right| \\
=\frac{1}{2 \sqrt{v(t)}}\left|\exp \left(-2 \int_{x}^{t} \sqrt{v(u)} d u\right)-1\right|
\end{array}
$$

and since with $x \leq t$ one has $\exp \left(-2 \int_{x}^{t} \sqrt{v(u)} d u\right) \leq 1$, we deduce that

$$
\left|C(x, t) \frac{\gamma_{0}(t, \lambda)}{\gamma_{0}(x, \lambda)}\right| \leq \frac{1}{\sqrt{v(t)}} .
$$

Hence

$$
\begin{aligned}
|R(x, t, \lambda)|=\left|C(x, t) \frac{\gamma_{0}(t, \lambda)}{\gamma_{0}(x, \lambda)}[(\lambda+q(t)) I-U(t)]\right| & \\
& \leq \frac{1}{\sqrt{v(t)}}(|\lambda|+|q(t)|+|U(t)|) .
\end{aligned}
$$

By virtue of (3)-(5), (7),

$$
\frac{1}{\sqrt{v(t)}}(|\lambda|+|q(t)|+|U(t)|) \in L(0, \infty)
$$

which implies that the integral equation has a unique solution $\chi(x, \lambda)$, and $|\chi(x, \lambda)| \leq$ const. By (18), one has that $\lim _{x \rightarrow \infty} \chi(x, \lambda)=I_{m}$, which already implies (11).

Differentiate (15) to get

$$
\frac{\Phi^{\prime}(x, \lambda)}{\gamma_{0}^{\prime}(x, \lambda)}=I_{m}+\int_{x}^{\infty} S(x, t, \lambda) \chi(t, \lambda) d t
$$

where

$$
S(x, t, \lambda)=K_{x}^{\prime}(x, t, \lambda) \frac{\gamma_{0}(t, \lambda)}{\gamma_{0}^{\prime}(x, \lambda)}=C_{x}^{\prime}(x, t) \frac{\gamma_{0}(t, \lambda)}{\gamma_{0}^{\prime}(x, \lambda)}\left[(\lambda+q(t)) I_{m}-U(t)\right] .
$$


Since

$$
\begin{gathered}
\left|C_{x}^{\prime}(x, t) \frac{\gamma_{0}(t, \lambda)}{\gamma_{0}^{\prime}(x, \lambda)}\right|=\left|\gamma_{0}^{2}(t) t \frac{\gamma_{\infty}^{\prime}(x, \lambda)}{\gamma_{0}^{\prime}(x, \lambda)}-\gamma_{\infty}(t, \lambda) \gamma_{0}(t, \lambda)\right| \\
=\frac{1}{2 \sqrt{v(t)}}\left|\exp \left(-2 \int_{0}^{t} \sqrt{v(u)} d u\right) \frac{\frac{1}{4} v^{-\frac{5}{4}} v^{\prime}-\sqrt{v}}{\frac{1}{4} v^{-\frac{5}{4}} v^{\prime}+\sqrt{v}} \exp \left(2 \int_{0}^{x} \sqrt{v(u)} d u\right)-1\right| \\
\leq \frac{1}{2 \sqrt{v(t)}}\left(\exp \left(-2 \int_{x}^{t} \sqrt{v(u)} d u\right)+1\right) \leq \frac{1}{\sqrt{v(t)}},
\end{gathered}
$$

one has

$$
|S(x, t, \lambda)| \leq \frac{1}{\sqrt{v(t)}}[|\lambda|+|q(t)|+|U(t)|] \in L(0, \infty) .
$$

Therefore, (21) now implies (12).

2. Denote by

$$
\widehat{\Psi}(x, \lambda)=\left(\begin{array}{cccc}
\Psi_{11}(x, \lambda) & \Psi_{12}(x, \lambda) & \ldots & \Psi_{1 r}(x, \lambda) \\
0 & \Psi_{22}(x, \lambda) & \ldots & \Psi_{2 r}(x, \lambda) \\
\ldots & \ldots & \ldots & \ldots \\
0 & 0 & \ldots & \Psi_{r r}(x, \lambda)
\end{array}\right)
$$

the matrix solution of (1) that increases at infinity. The diagonal blocks $\Psi_{k k}(x)$ are the $m_{k} \times m_{k}$-matrices with $m_{k} \geq 1, k=\overline{1, r}$. Equation (6) is equivalent to the integral equation

$$
\widehat{\Psi}(x, \lambda)=\gamma_{\infty}(x, \lambda) I_{m}-\int_{0}^{x} K(x, t, \lambda) \widehat{\Psi}(t, \lambda) d t,
$$

where, just as in $(15)$, the kernel $K(x, t, \lambda)$ is given by (16). Now set

$$
\chi(x, \lambda)=\frac{\widehat{\Psi}(x, \lambda)}{\gamma_{\infty}(x, \lambda)}
$$

to rewrite equation (22) in the form

$$
\chi(x, \lambda)=I_{m}-\int_{0}^{x} R(x, t, \lambda) \chi(t, \lambda) d t,
$$

where

$$
R(x, t, \lambda)=C(x, t) \frac{\gamma_{\infty}(t, \lambda)}{\gamma_{\infty}(x, \lambda)}\left[(\lambda+q(t)) I_{m}-U(t)\right] .
$$


Since $t \leq x$, one has

$$
\begin{array}{r}
\left|C(x, t) \frac{\gamma_{\infty}(t, \lambda)}{\gamma_{\infty}(x, \lambda)}\right|=\left|\gamma_{0}(t, \lambda) \gamma_{\infty}(t, \lambda)-\gamma_{\infty}^{2}(t, \lambda) \frac{\gamma_{0}(x, \lambda)}{\gamma_{\infty}(x, \lambda)}\right| \\
=\frac{1}{2 \sqrt{v(t)}}\left|1-\exp \left(-2 \int_{0}^{t} \sqrt{v(u)} d u\right) \exp \left(-2 \int_{0}^{x} \sqrt{v(u)} d u\right)\right| \\
=\frac{1}{2 \sqrt{v(t)}}\left(1-\exp \left(-2 \int_{t}^{x} \sqrt{v(u)} d u\right)\right) \leq \frac{1}{\sqrt{v(t)}}
\end{array}
$$

and therefore

$$
|R(x, t, \lambda)| \leq \frac{1}{\sqrt{v(t)}}[|\lambda|+|q(t)|+|U(t)|] \in L(0, \infty) .
$$

It follows that integral equation (23) has a unique solution $\chi(x, \lambda)$, and $|\chi(x, \lambda)| \leq$ const.

Pass in (23) to a limit as $x \rightarrow \infty$ to get

$$
\lim _{x \rightarrow \infty} \chi(x, \lambda)=I_{m}+\widetilde{C}(\lambda)
$$

where $\widetilde{C}(\lambda)$ is a triangular matrix, that is,

$$
\lim _{x \rightarrow \infty} \frac{\widehat{\Psi}(x, \lambda)}{\gamma_{\infty}(x, \lambda)}=I_{m}+\widetilde{C}(\lambda)
$$

Now consider another matrix solution that increases at infinity

$$
\widetilde{\Psi}(x, \lambda)=\left(\begin{array}{cccc}
\widetilde{\Psi}_{11}(x, \lambda) & \widetilde{\Psi}_{12}(x, \lambda) & \ldots & \widetilde{\Psi}_{1 r}(x, \lambda) \\
0 & \widetilde{\Psi}_{22}(x, \lambda) & \ldots & \widetilde{\Psi}_{2 r}(x, \lambda) \\
\ldots & \ldots & \ldots & \ldots \\
0 & 0 & \ldots & \widetilde{\Psi}_{r r}(x, \lambda)
\end{array}\right)
$$

where

$$
\widetilde{\Psi}_{k k}(x, \lambda)=\Phi_{k k}(x, \lambda) \int_{a}^{x} \Phi_{k k}^{-1}(t, \lambda)\left(\Phi_{k k}^{*}(t, \lambda)\right)^{-1} d t, \quad k=\overline{1, r}, \quad(a \geq 0),
$$

$\Phi_{k k}(x, \lambda)$ are the diagonal blocks of the matrix solution $\Phi(x, \lambda)$ as in Section 1. 
In view of (11) and the definitions of $\gamma_{0}(x, \lambda), \gamma_{\infty}(x, \lambda)$, one has

$$
\begin{aligned}
& \widetilde{\Psi}_{k k}(x, \lambda)=\gamma_{0}(x, \lambda)\left(I_{m_{k}}+o_{x}\left(I_{m_{k}}\right)\right) \int_{0}^{x} \frac{I_{m_{k}}+o_{t}\left(I_{m_{k}}\right)}{\gamma_{0}^{2}(t, \lambda)} d t \\
& =\gamma_{0}(x, \lambda) \int_{0}^{x} \frac{d t}{\gamma_{0}^{2}(t, \lambda)}\left(I_{m_{k}}+o\left(I_{m_{k}}\right)\right)=\gamma_{\infty}(x, \lambda)\left(I_{m_{k}}+o\left(I_{m_{k}}\right)\right) .
\end{aligned}
$$

Hence

$$
\lim _{x \rightarrow \infty} \frac{\widetilde{\Psi}_{k k}(x, \lambda)}{\gamma_{\infty}(x, \lambda)}=I_{m_{k}}, \quad k=\overline{1, r} .
$$

Since $\widehat{\Psi}(x, \lambda)$ and $\widetilde{\Psi}(x, \lambda)$ are the matrix solutions of (1) that increase at infinity,

$$
\widehat{\Psi}(x, \lambda)=\widetilde{\Psi}(x, \lambda)+\Phi(x, \lambda) C_{0}(\lambda),
$$

where $C_{0}(\lambda)$ is a block-triangular matrix. Additionally, one has

$$
\lim _{x \rightarrow \infty} \frac{\widehat{\Psi}(x, \lambda)}{\gamma_{\infty}(x, \lambda)}=\lim \frac{\widetilde{\Psi}(x, \lambda)}{\gamma_{\infty}(x, \lambda)} .
$$

Hence, by virtue of (26),

$$
\lim _{x \rightarrow \infty} \frac{\Psi_{k k}(x, \lambda)}{\gamma_{\infty}(x, \lambda)}=I_{m_{k}}, \quad k=\overline{1, m}
$$

and in (24) one has

$$
\widetilde{C}(\lambda)=\left(\begin{array}{cccc}
0 & C_{12}(\lambda) & \ldots & C_{1 r}(\lambda) \\
0 & 0 & \ldots & C_{2 r}(\lambda) \\
\ldots & \ldots & \ldots & \ldots \\
0 & 0 & \ldots & 0
\end{array}\right)
$$

The solution $\Psi(x, \lambda)$ given by $\Psi(x, \lambda)=\widehat{\Psi}(x, \lambda)(I+\widetilde{C}(\lambda))^{-1}$ is subject to condition (13).

Use (11), (12) to differentiate (27), then find the asymptotics of $\widetilde{\Psi}^{\prime}(x, \lambda)$ as $x \rightarrow \infty$ similarly to (25) to obtain (14). Theorem 1 is proved.

II. Now consider the case when $v(x)=x^{2 \alpha}, 0<\alpha \leq 1$. Suppose that the coefficients of equation (1) satisfy the condition

$$
\int_{0}^{\infty}|U(t)| t^{-\alpha} d t<\infty .
$$


Rewrite (1) in the form

$$
-\bar{y}^{\prime \prime}+\left(x^{2 \alpha}-\lambda+q(x, \lambda)\right) \bar{y}=\left(q(x, \lambda) I_{m}-U(x)\right) \bar{y},
$$

where $q(x, \lambda)$ is defined similarly to $q(x)$ in $(7)$ :

$$
q(x, \lambda)=\frac{5}{16}\left(\frac{v^{\prime}(x)}{v(x)-\lambda}\right)^{2}-\frac{1}{4} \frac{v^{\prime \prime}(x)}{v(x)-\lambda}
$$

i.e.,

$$
q(x, \lambda)=\frac{5 \alpha^{2}}{4}\left(\frac{x^{2 \alpha-1}}{x^{2 \alpha}-\lambda}\right)^{2}-\frac{\alpha(2 \alpha-1) x^{2 \alpha-2}}{2\left(x^{2 \alpha}-\lambda\right)} .
$$

Consider the functions

$$
\begin{aligned}
\gamma_{0}(x, \lambda) & =\frac{1}{\sqrt[4]{4\left(x^{2 \alpha}-\lambda\right)}} \exp \left(-\int_{a}^{x} \sqrt{u^{2 \alpha}-\lambda} d u\right), \\
\gamma_{\infty}(x, \lambda) & =\frac{1}{\sqrt[4]{4\left(x^{2 \alpha}-\lambda\right)}} \exp \left(\int_{a}^{x} \sqrt{u^{2 \alpha}-\lambda} d u\right)
\end{aligned}
$$

These solutions form a fundamental system of solutions for the scalar differential equation

$$
-z^{\prime \prime}+\left(x^{2 \alpha}-\lambda+q(x, \lambda)\right) z=0
$$

such that for all $x$

$$
W\left(\gamma_{0}, \gamma_{\infty}\right):=\gamma_{0}(x, \lambda) \gamma_{\infty}^{\prime}(x, \lambda)-\gamma_{0}^{\prime}(x, \lambda) \gamma_{\infty}(x, \lambda)=1
$$

We are about to establish the asymptotics ${ }^{\star}$ of $\gamma_{0}(x, \lambda)$ as $x \rightarrow \infty$ :

$$
\gamma_{0}(x, \lambda)=\left(2 x^{\alpha}\right)^{-\frac{1}{2}}\left(1-\frac{\lambda}{x^{2 \alpha}}\right)^{-\frac{1}{4}} \exp \left(-\int_{a}^{x} u^{\alpha}\left(1-\frac{\lambda}{u^{2 \alpha}}\right)^{\frac{1}{2}} d u\right)
$$

After expanding here the integrand, we obtain the exponential as follows:

$$
\exp \left(-\int_{a}^{x} u^{\alpha}\left(1-\frac{1}{2} \frac{\lambda}{u^{2 \alpha}}-\sum_{k=2}^{\infty} \frac{1 \cdot 3 \cdot \ldots \cdot(2 k-3)}{k ! 2^{k}}\left(\frac{\lambda}{u^{2 \alpha}}\right)^{k}\right) d u\right) .
$$

${ }^{\star}$ With $\alpha=1$ and $\alpha=\frac{1}{2}$, that is, with $v(x)=x^{2}$ and $v(x)=x$, the asymptotics of $\gamma_{0}(x, \lambda)$ and $\gamma_{\infty}(x, \lambda)$ are already known. See the monograph [12], where the Langer method is applied to obtain the asymptotics in a different form by using the Hankel function. 
In the case $\frac{\alpha+1}{2 \alpha}=n \in \mathbb{N}$, i.e., $\alpha=\frac{1}{2 n-1}$, after integration this expression has the form

$$
\begin{aligned}
& c \exp \left(-\frac{x^{1+\alpha}}{1+\alpha}\right.+\frac{\lambda}{2} \frac{x^{1-\alpha}}{1-\alpha} \\
&\left.+\sum_{k=2}^{n-1} \frac{1 \cdot 3 \cdot \ldots \cdot(2 k-3)}{k !}\left(\frac{\lambda}{2}\right)^{k} \frac{x^{1-(2 k-1) \alpha}}{1-(2 k-1) \alpha}\right) \\
& \times \exp \left(\frac{1 \cdot 3 \cdot \ldots \cdot(2 n-3)}{n !}\left(\frac{\lambda}{2}\right)^{n} \ln x+o(1)\right) \\
&=c \exp \left(-\frac{x^{1+\alpha}}{1+\alpha}+\frac{\lambda}{2} \frac{x^{1-\alpha}}{1-\alpha}\right. \\
&\left.+\sum_{k=2}^{n-1} \frac{1 \cdot 3 \cdot \ldots \cdot(2 k-3)}{k !}\left(\frac{\lambda}{2}\right)^{k} \frac{x^{1-(2 k-1) \alpha}}{1-(2 k-1) \alpha}\right) x^{\frac{1 \cdot 3 \cdot \ldots \cdot(2 n-3)}{n !}\left(\frac{\lambda}{2}\right)^{n}}(1+o(1)) .
\end{aligned}
$$

The asymptotics of $\gamma_{0}(x, \lambda)$ as $x \rightarrow \infty$ is as follows:

$$
\begin{array}{r}
\gamma_{0}(x, \lambda)=c \exp \left(-\frac{x^{1+\alpha}}{1+\alpha}+\frac{\lambda}{2} \frac{x^{1-\alpha}}{1-\alpha}\right. \\
\left.+\sum_{k=2}^{n-1} \frac{1 \cdot 3 \cdot \ldots \cdot(2 k-3)}{k !}\left(\frac{\lambda}{2}\right)^{k} \frac{x^{1-(2 k-1) \alpha}}{1-(2 k-1) \alpha}\right) x^{\frac{1 \cdot 3 \cdots \cdot(2 n-3)}{n !}\left(\frac{\lambda}{2}\right)^{n}-\frac{\alpha}{2}} \\
\times(1+o(1)) .
\end{array}
$$

With $\alpha=1(n=1), \gamma_{0}(x, \lambda)$ has the following asymptotics at infinity:

$$
\gamma_{0}(x, \lambda)=c x^{\frac{\lambda-1}{2}} \exp \left(-\frac{x^{2}}{2}\right)(1+o(1)) .
$$

In the case $\frac{\alpha+1}{2 \alpha} \notin \mathbb{N}$, set $n=\left[\frac{\alpha+1}{2 \alpha}\right]+1$, with $[\beta]$ being the integral part of $\beta$, to obtain the following asymptotics for $\gamma_{0}(x, \lambda)$ at infinity:

$$
\begin{aligned}
\gamma_{0}(x, \lambda)=c x^{-\frac{\alpha}{2}} & \exp \left(-\frac{x^{1+\alpha}}{1+\alpha}+\frac{\lambda}{2} \frac{x^{1-\alpha}}{1-\alpha}\right. \\
& \left.+\sum_{k=2}^{n-1} \frac{1 \cdot 3 \cdot \ldots \cdot(2 k-3)}{k !}\left(\frac{\lambda}{2}\right)^{k} \frac{x^{1-(2 k-1) \alpha}}{1-(2 k-1) \alpha}\right) \\
& \times \exp \left(-\frac{1 \cdot 3 \cdot \ldots \cdot(2 n-3)}{n !}\left(\frac{\lambda}{2}\right)^{n} \frac{x^{-\alpha}}{\alpha}\right)\left(1+o\left(x^{-\alpha}\right)\right) .
\end{aligned}
$$


In particular, with $\alpha=\frac{1}{2}(n=2)$ one has

$$
\gamma_{0}(x, \lambda)=c x^{-\frac{1}{4}} \exp \left(-\frac{2}{3} x^{\frac{3}{2}}+\lambda x^{\frac{1}{2}}-\left(\frac{\lambda}{2}\right)^{2} x^{-\frac{1}{2}}\right)\left(1+o\left(x^{-\frac{1}{2}}\right)\right) .
$$

A similar procedure allows one to establish the asymptotics of $\gamma_{\infty}(x, \lambda)$ as $x \rightarrow \infty$. If $\frac{\alpha+1}{2 \alpha}=n \in \mathbb{N}$, i.e., $\alpha=\frac{1}{2 n-1}$, then

$$
\begin{aligned}
& \gamma_{\infty}(x, \lambda)=c \exp \left(\frac{x^{1+\alpha}}{1+\alpha}-\frac{\lambda}{2} \frac{x^{1-\alpha}}{1-\alpha}\right. \\
&\left.-\sum_{k=2}^{n-1} \frac{1 \cdot 3 \cdot \ldots \cdot(2 k-3)}{k !}\left(\frac{\lambda}{2}\right)^{k} \frac{x^{1-(2 k-1) \alpha}}{1-(2 k-1) \alpha}\right) x^{-\left(\frac{1 \cdot 3 \cdots \cdot(2 n-3)}{n !}\left(\frac{\lambda}{2}\right)^{n}+\frac{\alpha}{2}\right)} \\
& \times(1+o(1)) .
\end{aligned}
$$

With $\alpha=1(n=1)$, this becomes

$$
\gamma_{\infty}(x, \lambda)=c x^{-\frac{\lambda+1}{2}} \exp \left(\frac{x^{2}}{2}\right)(1+o(1))
$$

In the case $\frac{\alpha+1}{2 \alpha} \notin \mathbb{N}$, we set $n=\left[\frac{\alpha+1}{2 \alpha}\right]+1$ to get the asymptotics

$$
\begin{aligned}
\gamma_{\infty}(x, \lambda)=c x^{-\frac{\alpha}{2}} & \exp \left(\frac{x^{1+\alpha}}{1+\alpha}-\frac{\lambda}{2} \frac{x^{1-\alpha}}{1-\alpha}\right. \\
& \left.-\sum_{k=2}^{n-1} \frac{1 \cdot 3 \cdot \ldots \cdot(2 k-3)}{k !}\left(\frac{\lambda}{2}\right)^{k} \frac{x^{1-(2 k-1) \alpha}}{1-(2 k-1) \alpha}\right) \\
& \quad \times \exp \left(\frac{1 \cdot 3 \cdot \ldots \cdot(2 n-3)}{n !}\left(\frac{\lambda}{2}\right)^{n} \frac{x^{-\alpha}}{\alpha}\right)\left(1+o\left(x^{-\alpha}\right)\right) .
\end{aligned}
$$

In the case $\alpha=\frac{1}{2}(n=2)$, one has

$$
\gamma_{\infty}(x, \lambda)=c x^{-\frac{1}{4}} \exp \left(\frac{2}{3} x^{\frac{3}{2}}-\lambda x^{\frac{1}{2}}+\left(\frac{\lambda}{2}\right)^{2} x^{-\frac{1}{2}}\right)\left(1+o\left(x^{-\frac{1}{2}}\right)\right) .
$$

Theorem 2. With $0<\alpha \leq 1$ and under condition (28), the claim of Theorem 1 is valid for equation (1). 
The proof goes similarly to that of Theorem 1. It should be observed in this context that

$$
\begin{gathered}
\left|C(x, t, \lambda) \frac{\gamma_{0}(t, \lambda)}{\gamma_{0}(x, \lambda)}\right|=\left|\gamma_{0}^{2}(t, \lambda) \frac{\gamma_{\infty}(x, \lambda)}{\gamma_{0}(x, \lambda)}-\gamma_{0}(t, \lambda) \gamma_{\infty}(t, \lambda)\right| \\
=\left|\frac{1}{2 \sqrt{t^{2 \alpha}-\lambda}} \exp \left(-2 \int_{a}^{t} \sqrt{u^{2 \alpha}-\lambda} d u\right) \exp \left(2 \int_{a}^{x} \sqrt{u^{2 \alpha}-\lambda} d u\right)-\frac{1}{2 \sqrt{t^{2 \alpha}-\lambda}}\right| \\
=\frac{1}{2 \sqrt{t^{2 \alpha}-\lambda}}\left|\exp \left(-2 \int_{x}^{t} \sqrt{u^{2 \alpha}-\lambda} d u-1\right)\right| .
\end{gathered}
$$

As $x \leq t$, one has $\exp \left(-2 \int_{x}^{t} \sqrt{u^{2 \alpha}-\lambda} d u\right) \leq 1$, hence

$$
\left|C(x, t, \lambda) \frac{\gamma_{0}(t, \lambda)}{\gamma_{0}(x, \lambda)}\right| \leq \frac{1}{\sqrt{t^{2 \alpha}-\lambda}}
$$

and thus

$$
\begin{aligned}
|R(x, t, \lambda)| & =\left|C(x, t, \lambda) \frac{\gamma_{0}(t, \lambda)}{\gamma_{0}(x, \lambda)}\left[q(t, \lambda) I_{m}-U(t)\right]\right| \\
& \leq \frac{1}{\sqrt{t^{2 \alpha}-\lambda}}(|q(t, \lambda)|+|U(t)|) .
\end{aligned}
$$

It follows from (28) and (30) that

$$
\frac{1}{\sqrt{t^{2 \alpha}-\lambda}}(|q(t, \lambda)|+|U(t)|) \in L(a, \infty) .
$$

Hence integral equation (18) has a unique solution $\chi(x, \lambda)$ and $|\chi(x, \lambda)| \leq$ const. By (18), we have that $\lim _{x \rightarrow \infty} \chi(x, \lambda)=I$, which implies (11).

The rest of the claims of Theorem 1 can be obtained in a similar way.

Theorem 1, together with asymptotic formulas (33) and (37), allows one to deduce

Corollary 1. With $\alpha=1$, i.e., $v(x)=x^{2}$, under condition (28), equation (1) has a unique $m \times m$ matrix solution $\Phi(x, \lambda)$, which decreases at infinity, satisfies the conditions

$$
\lim _{x \rightarrow \infty} \frac{\Phi(x, \lambda)}{\gamma_{0}(x, \lambda)}=I_{m}
$$

and

$$
\lim _{x \rightarrow \infty} \frac{\Phi^{\prime}(x, \lambda)}{\gamma_{0}^{\prime}(x, \lambda)}=I_{m}
$$


where $\gamma_{0}(x, \lambda)=x^{\frac{\lambda-1}{2}} \exp \left(-\frac{x^{2}}{2}\right)$. Also, the equation has a unique $m \times m$ matrix solution $\Psi(x, \lambda)$, which increases at infinity, satisfies the conditions

$$
\lim _{x \rightarrow \infty} \frac{\Psi(x, \lambda)}{\gamma_{\infty}(x, \lambda)}=I_{m}
$$

and

$$
\lim _{x \rightarrow \infty} \frac{\Psi^{\prime}(x, \lambda)}{\gamma_{\infty}^{\prime}(x, \lambda)}=I_{m}
$$

where $\gamma_{\infty}(x, \lambda)=x^{-\frac{\lambda+1}{2}} \exp \left(\frac{x^{2}}{2}\right)$.

Theorem 2, together with asymptotic formulas (35) and (39), implies

Corollary 2. If $\alpha=\frac{1}{2}$, i.e., $v(x)=x$, under conditions (28), equation (1) has a unique $m \times m$ matrix solution $\Phi(x, \lambda)$, which decreases at infinity, satisfies the conditions

$$
\lim _{x \rightarrow \infty} \frac{\Phi(x, \lambda)}{\gamma_{0}(x, \lambda)}=I_{m}
$$

and

$$
\lim _{x \rightarrow \infty} \frac{\Phi^{\prime}(x, \lambda)}{\gamma_{0}^{\prime}(x, \lambda)}=I_{m}
$$

where $\gamma_{0}(x, \lambda)=x^{-\frac{1}{4}} \exp \left(-\frac{2}{3} x^{\frac{3}{2}}+\lambda x^{\frac{1}{2}}\right)$. Also, the equation has an $m \times m$ matrix solution $\Psi(x, \lambda)$ which increases at infinity, satisfies the conditions

$$
\lim _{x \rightarrow \infty} \frac{\Psi(x, \lambda)}{\gamma_{\infty}(x, \lambda)}=I_{m}
$$

and

$$
\lim _{x \rightarrow \infty} \frac{\Psi^{\prime}(x, \lambda)}{\gamma_{\infty}^{\prime}(x, \lambda)}=I_{m},
$$

where $\gamma_{\infty}(x, \lambda)=x^{-\frac{1}{4}} \exp \left(\frac{2}{3} x^{\frac{3}{2}}-\lambda x^{\frac{1}{2}}\right)$.

$\mathrm{R}$ e $\mathrm{m}$ a $\mathrm{r} \mathrm{k}$ 1. In the monograph [14], it was shown that the scalar equation

$$
-\phi^{\prime \prime}+x^{2} \phi=\lambda \phi,
$$

with $\lambda=2 n+1$, has a solution

$$
\phi_{n}(x)=H_{n}(x) \exp \left(-\frac{x^{2}}{2}\right),
$$


where $H_{n}(x)$ is the Chebishev-Hermit polynomial. Note that this polynomial has the following asymptotics as $x \rightarrow \infty: H_{n}(x)=(2 x)^{n}(1+o(1))$, and thus the asymptotics of the solution $\phi_{n}(x)$ of (43) as $x \rightarrow \infty$ is

$$
\phi_{n}(x)=(2 x)^{n} \exp \left(-\frac{x^{2}}{2}\right) \cdot(1+o(1)) .
$$

In the case when in (2) one has $U(x)=0, v(x)=x^{2}$, matrix equation (1) splits into $m$ scalar equations of the form (43). The matrix solution $\Phi(x, \lambda)$ in this case appears to be diagonal. Denote by $\phi(x, \lambda)$ the diagonal elements of the matrix $\Phi(x, \lambda)$. Then by virtue of $(42)$, the solution $\phi(x, \lambda)$ has the following asymptotics at infinity:

$$
\phi(x, \lambda)=(x)^{\frac{\lambda-1}{2}} \exp \left(-\frac{x^{2}}{2}\right)(1+o(1)) .
$$

In particular, with $\lambda=2 n+1$ this allows one to derive a solution which is a scalar multiple of $\phi_{n}(x)$.

III. Suppose at $x=0$, we are given the boundary condition

$$
B y^{\prime}(0)-C y(0)=0,
$$

where $B$ and $C$ are the commuting block-triangular matrices of the same structure as the coefficients of the differential equation

$$
B=\left(\begin{array}{cccc}
B_{11} & B_{12} & \ldots & B_{1 r} \\
0 & B_{22} & \ldots & B_{2 r} \\
\ldots & \ldots & \ldots & \ldots \\
0 & 0 & \ldots & B_{r r}
\end{array}\right), \quad C=\left(\begin{array}{cccc}
C_{11} & C_{12} & \ldots & C_{1 r} \\
0 & C_{22} & \ldots & C_{2 r} \\
\ldots & \ldots & \ldots & \ldots \\
0 & 0 & \ldots & C_{r r}
\end{array}\right)
$$

$B_{k k}, C_{k k}, k=\overline{1, r}$, are $m_{k} \times m_{k}$ Hermitian matrices and $m_{k} \geq 1, \sum_{k=1}^{r} m_{k}=m$, which satisfy the conditions $\operatorname{det}\left(B^{2}+C^{2}\right)=\prod_{k=1}^{r} \operatorname{det}\left(B_{k k}^{2}+C_{k k}^{2}\right) \neq 0$. It follows from $B C=C B$ that $B_{k k} C_{k k}=C_{k k} B_{k k}, k=\frac{k=1}{1, r}$.

Lemma 1. The boundary condition (46) can be rewritten in the equivalent form:

$$
\cos A \bar{y}^{\prime}(0)-\sin A \bar{y}(0)=0,
$$

where $A$ is a block-triangular matrix of the same structure as the matrices $B, C$ in (47). 
In fact, since $B$ and $C$ commute, each of the matrices commutes with the matrix $\left(B^{2}+C^{2}\right)$ as well as with the matrix $\left(B^{2}+C^{2}\right)^{-\frac{1}{2}}$. It follows that there exists a block-triangular matrix $A$ such that

$$
\cos A=\left(B^{2}+C^{2}\right)^{-\frac{1}{2}} B, \quad \sin A=\left(B^{2}+C^{2}\right)^{-\frac{1}{2}} C .
$$

After multiplying on the left by $\left(B^{2}+C^{2}\right)^{-\frac{1}{2}}$, the boundary condition (46) has the form of (48).

Together with problem (1) with boundary condition (48), consider the split system

$$
l_{k}\left[\bar{y}_{k}\right]=-\bar{y}_{k}^{\prime \prime}+\left(v(x) I_{m_{k}}+U_{k k}(x)\right) \bar{y}_{k}=\lambda \bar{y}_{k}, \quad k=\overline{1, r},
$$

with the boundary conditions

$$
\cos A_{k k} \cdot \bar{y}_{k}^{\prime}(0)-\sin A_{k k} \cdot \bar{y}_{k}(0)=0, \quad k=\overline{1, r},
$$

where $A_{k k}$ are the diagonal elements of the matrix $A, A_{k k}, k=\overline{1, r}$ are $m_{k} \times m_{k}$ Hermitian matrices with $m_{k} \geq 1, \sum_{k=1}^{r} m_{k}=m$.

Denote by $L_{0}$ the minimal differential operator generated by the differential expression $l[\bar{y}]$ and boundary condition (48). Let also $L_{k}, k=\overline{1, r}$, stand for the minimal symmetric operators in $L_{2}\left(H_{m_{k}},(0, \infty)\right)$ generated by the differential expressions $l_{k}\left[\bar{y}_{k}\right]$ and boundary conditions (49). By the assumptions on the coefficients, along with the relatively small amount of the perturbations $U_{k k}(x)$ $\left(|U(x)| \cdot v^{-1}(x) \rightarrow 0\right.$ as $x \rightarrow \infty$ or $\left.|U| \cdot v^{-1} \in L^{\infty}(\mathbb{R})\right)$, we are in the case of a limit point at infinity for each symmetric operator $L_{k}, k=\overline{1, r}$. Hence their selfadjoint extensions ${ }^{\star} \widetilde{L}_{k}$ are given by the closures of $L_{k}$. The operators $\widetilde{L}_{k}$ are semibounded, and their spectra are discrete.

Denote by $L$ the extension of the operator $L_{0}$ given by the requirement that the functions from the domain of $L$ are in $L_{2}\left(H_{m},(0, \infty)\right)$.

Theorem 3. Consider equation (1). If either conditions (3), (4), (5) with $\alpha>1$ or condition (28) with $0<\alpha \leq 1$ are satisfied, then the discrete spectrum of $L$ is real and coincides with the union of spectra of the selfadjoint operators $\widetilde{L}_{k}, k=\overline{1, r}$, i.e.,

$$
\sigma_{d}(L)=\bigcup_{k=1}^{r} \sigma\left(\widetilde{L}_{k}\right) .
$$

${ }^{\star}$ The selfadjointness property for the general (4-term) Sturm-Liouville differential equations with matrix coefficients was studied in [24]. 
P r o o f. Recall that the matrix solutions $\Phi(x, \lambda), \Psi(x, \lambda)$, as in Theorems 1,2 , form a fundamental system of solutions of (1). It follows that every vector solution $\bar{y}(x, \lambda)$ of $(1)$ is representable in the form

$$
\bar{y}(x, \lambda)=\Phi(x, \lambda) \bar{h}+\Psi(x, \lambda) \bar{g},
$$

with $\bar{h}$ and $\bar{g}$ being constant vectors. A solution $\bar{y}(x, \lambda) \in L_{2}\left(H_{m},(0, \infty)\right)$ if and only if $\bar{g}=0$, that is,

$$
\bar{y}(x, \lambda)=\Phi(x, \lambda) \bar{h} .
$$

The eigenvalues of $L$ coincide with zeros of the determinant $\Delta(\lambda):=\operatorname{det} \Omega(0, \lambda)$, where

$$
\Omega(0, \lambda)=\cos A \Phi^{\prime}(0, \lambda)-\sin A \Phi(0, \lambda) .
$$

Since the matrices $A, \Phi(0, \lambda), \Phi^{\prime}(0, \lambda)$ are block-triangular, one has $\Delta(\lambda)=$ $\prod_{k=1}^{r} \Delta_{k}(\lambda)$, where $\Delta_{k}(\lambda):=\operatorname{det} \Omega_{k}(0, \lambda)$,

$$
\Omega_{k}(0, \lambda)=\cos A_{k k} \Phi_{k k}^{\prime}(0, \lambda)-\sin A_{k k} \Phi_{k k}(0, \lambda) .
$$

On the other hand, zeros of $\Delta_{k}(\lambda), k=\overline{1, r}$, (cf. (49)) are the eigenvalues of the selfadjoint operator $\widetilde{L}_{k}$ and hence are real. It follows that the discrete spectrum of $L$ is real and coincides with the union of spectra of the operators $\widetilde{L}_{k}$, $k=\overline{1, r}$. The Theorem is proved.

$\mathrm{R}$ e $\mathrm{m}$ a $\mathrm{rk} 2$. In the case $\alpha=1$ under the absence of condition (28), the claim of Theorem 3 can fail to be true.

$\mathrm{E} \times \mathrm{a} \mathrm{m} \mathrm{ple} 1$. Consider the equation

$$
l[\bar{y}]=-\bar{y}^{\prime \prime}+\left(\begin{array}{cc}
x^{2} & q(x) \\
0 & \pi^{2} x^{2}
\end{array}\right) \bar{y}=\lambda \bar{y}, \quad 0 \leq x<\infty, \quad \bar{y}=\left(\begin{array}{l}
y_{1} \\
y_{2}
\end{array}\right),
$$

with the boundary condition

$$
\bar{y}(0)=0 .
$$

The eigenvalues of the operators $\widetilde{L}_{1}$ and $\widetilde{L}_{2}$ generated by problem (52), (53) are not the same. Let $\lambda_{0}$ be an eigenvalue of $\widetilde{L}_{2}$, and $y_{2}\left(x, \lambda_{0}\right)$ be the corresponding eigenfunction. $\lambda_{0}$ is an eigenvalue of $L$ in the case when the solution $y_{1}\left(x, \lambda_{0}\right)$ of the equation $-y_{1}^{\prime \prime}+x^{2} y_{1}+q(x) y_{2}=\lambda_{0} y_{1}$, which satisfies the initial conditions $y_{1}(0, \lambda)=y_{1}^{\prime}(0, \lambda)=0$, is in $L_{2}(0 ; \infty)$. This solution is given by

$$
y_{1}\left(x, \lambda_{0}\right)=\int_{0}^{x} q(t) C\left(x, t, \lambda_{0}\right) y_{2}\left(t, \lambda_{0}\right) d t
$$


where $C\left(x, t, \lambda_{0}\right)$ is the Cauchy function of equation (43). With the coefficient $q(x)=y_{2}(x) e^{x^{\mu}}$ and $\mu>2$, one can prove (to be done in a subsequent work) that the integral $\int_{0}^{\infty} y_{1}^{2}\left(x, \lambda_{0}\right) d x$ diverges and hence $\lambda_{0} \notin \sigma(L)$.

It is to be shown in the separate paper that the (set of) poles of the Green function $G(x, t, \lambda)$ of $L$ coincide with $\sigma\left(\widetilde{L}_{1}\right) \cup \sigma\left(\widetilde{L}_{2}\right)$. If some $\lambda_{0} \in \sigma\left(\widetilde{L}_{1}\right)$, then $\lambda_{0} \in \sigma(L)$. On the other hand, $\lambda_{0} \in \sigma\left(\widetilde{L}_{2}\right)$ is an eigenvalue of $L$ if and only if $y_{1}\left(x, \lambda_{0}\right) \in L_{2}(0, \infty)$. If $\lambda_{0} \in \sigma\left(\widetilde{L}_{2}\right)$ and $y_{1}\left(x, \lambda_{0}\right) \notin L_{2}(0, \infty)$ as in Example 1, then $\lambda_{0}$ is a pole of the Green function $G(x, t, \lambda)$ but not an eigenvalue. These $\lambda_{0}$ are called the spectral singularities of the operator $L$. The special features of these points were initially discovered by M.A. Naimark in [15]. The term 'spectral singularity' was coined later on by J. Schwartz [16] (see also the monograph by M.A. Naimark [17], Supplement I by V.E. Ljance [18]).

IV. Suppose that in (1) one has $v(x) \equiv 0$, inf $U_{k k}(x) \rightarrow+\infty$ as $x \rightarrow \infty$ for all $k$, and $U_{j k}(x)$ with $j \neq k$ are compactly supported matrix valued functions whose carrier is $[0, b]$.

Denote by $\Phi_{k}(x, \lambda), \Theta_{k}(x, \lambda)$ the matrix solutions of the equation

$$
l_{k}\left[\bar{y}_{k}\right]=-\bar{y}_{k}^{\prime \prime}+U_{k k}(x) \bar{y}_{k}=\lambda \bar{y}_{k}, \quad k=\overline{1, r},
$$

which satisfy the initial conditions

$$
\begin{array}{llrl}
\Phi_{k}(0, \lambda) & =0, & \Phi_{k}^{\prime}(0, \lambda) & =I_{m_{k}}, \\
\Theta_{k}(0, \lambda) & =-I_{m_{k}}, & \Theta_{k}^{\prime}(0, \lambda) & =0 .
\end{array}
$$

For all nonreal $\lambda$ there exists a matrix Weyl function ${ }^{\star} M_{k}(\lambda)$ such that for any $\bar{h}_{k} \in H_{m_{k}}$ the solution

$$
\left(\Theta_{k}(x, \lambda)+\Phi_{k}(x, \lambda) M_{k}(\lambda)\right) \bar{h}_{k}
$$

of equation (54) is in $L_{2}\left(H_{m_{k}},(0, \infty)\right)$. The function $M_{k}(\lambda)$ is meromorphic, with its poles being on the real axis. These poles are just the points of spectrum of $\widetilde{L}_{k}$ generated in $L_{2}\left(H_{m_{k}},(0, \infty)\right)$ by the differential expression $l_{k}\left[\bar{y}_{k}\right]$ and the boundary condition $\bar{y}_{k}(0, \lambda)=0$. The matrix valued meromorphic function $M_{k}(\lambda)$ admits a representation in the form

$$
M_{k}(\lambda)=\mathfrak{M}_{k}(\lambda)\left(\mathfrak{N}_{k}(\lambda)\right)^{-1},
$$

${ }^{\star}$ The theory of matrix Weyl functions is a subject for a large number of papers. An extended bibliography on this topic can be found in the monograph [10]. Here we restrict ourselves to referring to [19]-[23]. 
where $\mathfrak{M}_{k}(\lambda), \mathfrak{N}_{k}(\lambda)$ are entire matrix valued functions of $\lambda$. In fact, all the matrix elements $m_{i j}^{(k)}(\lambda)$ of $M_{k}(\lambda)$ can be represented as ratios of entire functions with no common zeros

$$
m_{i j}^{(k)}(\lambda)=\frac{\mu_{i j}^{(k)}(\lambda)}{\nu_{i j}^{(k)}(\lambda)} .
$$

We choose $\mathfrak{N}_{k}(\lambda)=\left(n_{i j}^{(k)}(\lambda)\right)_{i, j=1}^{m_{k}}$ to be a diagonal matrix with $n_{j j}^{(k)}(\lambda)=$ $\prod_{i=1}^{m_{k}} \nu_{i j}^{(k)}(\lambda)$. In this case, the matrix $\mathfrak{M}_{k}(\lambda)$ is also an entire function of $\lambda$.

Then the function

$$
Y_{k}(x, \lambda)=\Theta_{k}(x, \lambda) \mathfrak{N}_{k}(\lambda)+\Phi_{k}(x, \lambda) \mathfrak{M}_{k}(\lambda)
$$

is a matrix solution of equation (54). For any vector $\bar{h}_{k} \in H_{m_{k}}$, the vector function $\bar{y}_{k}(x, \lambda)=Y_{k}(x, \lambda) \bar{h}_{k} \in L_{2}\left(H_{m_{k}},(0, \infty)\right)$ and the matrix valued function $Y_{k}(x, \lambda)$ are entire analytic in $\lambda$ on the complex plane at every $x \in[0, \infty)$.

The matrix

$$
Y(x, \lambda)=\left(\begin{array}{cccc}
Y_{1}(x, \lambda) & 0 & \ldots & 0 \\
0 & Y_{2}(x, \lambda) & \ldots & 0 \\
\ldots & \ldots & \ldots & \ldots \\
0 & 0 & \ldots & Y_{r}(x, \lambda)
\end{array}\right)
$$

is a solution of equation (1) for all $x \geq b$; given any vector $\bar{h} \in H_{m}$, one has $\bar{y}(x, \lambda)=Y(x, \lambda) \bar{h} \in L_{2}\left(H_{m},(0, \infty)\right)$. Denote by $\Phi(x, \lambda)$ the matrix solution of equation (1) which satisfies the following initial condition at $x=b$ :

$$
\Phi(b, \lambda)=Y(b, \lambda), \quad \Phi^{\prime}(b, \lambda)=Y^{\prime}(b, \lambda) .
$$

$\Phi(x, \lambda)$ is a solution of equation (1) for all $x \in[0, \infty)$. It has a block-triangular form

$$
\Phi(x, \lambda)=\left(\begin{array}{cccc}
\Phi_{11}(x, \lambda) & \Phi_{12}(x, \lambda) & \ldots & \Phi_{1 r}(x, \lambda) \\
0 & \Phi_{21}(x, \lambda) & \ldots & \Phi_{2 r}(x, \lambda) \\
\ldots & \ldots & \ldots & \ldots \\
0 & 0 & \ldots & \Phi_{r r}(x, \lambda)
\end{array}\right) \equiv Y(x, \lambda) \text { with } x \geq b
$$

Hence, for any vector $\bar{h}$ one has $\bar{y}(x, \lambda)=\Phi(x, \lambda) \bar{h} \in L_{2}\left(H_{m},(0, \infty)\right)$. This solution $\Phi(x, \lambda)$ is an entire analytic function of $\lambda$.

The eigenvalues of $L$ coincide with zeros of the determinant $\Delta(\lambda):=\operatorname{det} \Omega(0, \lambda)$, with the matrix $\Omega(0, \lambda)$ being given by $(51)$.

Now we are in a position to reproduce the final part of the proof of Theorem 3 in order to deduce 
Theorem 4. Suppose in (1) we have $v(x) \equiv 0$, inf $U_{k k}(x) \rightarrow+\infty$ as $x \rightarrow \infty$ for all $k$, and $U_{j k}(x)(j \neq k)$ are compactly supported functions whose carrier is $[0, b]$. Then the spectrum of $L$ is discrete, real, and coincides with the union of spectra of the operators $\widetilde{L}_{k}, k=\overline{1, r}$.

\section{References}

[1] A.M. Kholkin and F.S. Rofe-Beketov, Sturm Type Oscillation Theorems for Equations with Block-Triangular Matrix Coefficients. - Methods Funct. Anal. Topology 18 (2012), No. 2, 176-188.

[2] E.I. Bondarenko and F.S. Rofe-Beketov, Inverse Scattering Problem on the Semiaxis for a System with a Triangular Matrix Potential. - Math. Phys., Anal., Geom. 10, (2003), No. 3, 412-424. (Russian)

[3] E.I. Bondarenko and F.S. Rofe-Beketov, Phase Equivalent Matrix Potential. - Electromagnetic waves and electronic systems 5 (2000), No. 3, 6-24. (Russian) (Engl. transl.: Telecommun. Radio Eng. 56 (2001), Nos. 8 and 9, 4-29).

[4] F.S. Rofe-Beketov and E.I. Zubkova, Inverse Scattering Problem on the Axis for the Triangular $2 \times 2$ Matrix Potential with or without a Virtual Level. - Azerbaijan J. Math. 1 (2011), No. 2, 3-69.

[5] V.A. Marchenko, Spectral Theory of Sturm-Liouville Operators. Naukova Dumka, Kiev, 1972. (Russian)

[6] V.A. Marchenko, Sturm-Liouville Operators and its Applications. Naukova Dumka, Kiev, 1977. (Russian) (Engl. transl.: Oper. Theory Adv. Appl. 22 (1986), Birkhauser Verlag, Basel, xii+367pp.; revised edition AMS Chelsea Publishing, Providence R.I., 2011, xiv+396 pp.).

[7] V.A. Marchenko and F.S. Rofe-Beketov, Expansion in Eigenfunctions of Nonselfadjoint Singular Differential Operators. - Dokl. Akad. Nauk. SSSR 120 (1958), No. 5, 963-966. (Russian)

[8] F.S. Rofe-Beketov, Expansion in Eigenfunctions of Infinite Systems of Differential Equations in the Non-selfadjoint and Selfadjoint Cases. - Mat. Sb. 51 (1960), No. 3, 293-342. (Russian)

[9] Z.S. Agranovich and V.A. Marchenko, The Inverse Problem of Scattering Theory. Kharkov State University, Kharkov, 1960. (Russian) (Engl. transl.: New YorkLondon, Gordon \& Breach, 1963).

[10] F.S. Rofe-Beketov and A.M. Kholkin, Spectral Analysis of Differential Operators. Interplay between Spectral and Oscillatory Properties, With Foreword by V.A. Marchenko, World Scientific, New Jersey, London, Singapore, Beijing, Shanghai, Hong Kong, Taipei, Chennai, 2005.

[11] A. Eremenko and A. Gabrielov, Spectral Loci of Sturm-Liouville Operators with Polynomial Potentials, in print. 
[12] E.Ch. Titchmarsh, Eigenfunction Expansions Associated with Second-Order Differential Equations, Vol. 2, Clarendon Press, Oxford, 1958. (Russian transl.: Vol. 2, Izdat. Inostran. Lit., Moscow, 1961).

[13] F.S. Rofe-Beketov and E.Kh. Khristov, Asymptotic and Analytic Questions Connected with Scattering by a Highly Singular Potential, Collection of Proceeding of Fiz. Tekh. Inst. Niz. Temp. Akad. Nauk Ukrain. SSR. Mathematics, Physics and Functional Analysis Series, Kharkov, 1971, No. 2, 122-168. (Russian)

[14] A.N. Tichonov and A.A. Samarsky, Equations of Mathematical Physics. Nauka, Moscow, 1972. (Russian)

[15] M.A. Naimark, Research of Spectrum and Expansion in Eigenfunctions of Nonselfadjoint Differential Operators of Second Order on the Semiaxis. - Tr. Mosk. Mat. Obs., 3 (1954), 181-270. (Russian)

[16] J.T. Schwartz, Some Non-selfadjoint Operators. - Comm. Pure Appl. Math. XIII (1960), 609-639.

[17] M.A. Naimark, Linear Differential Operators. Nauka, Moscow, 1969. (Russian) (Engl. transl.: Frederic Ungar Publishing Co., New York, Part I 1967, Part II 1968.)

[18] V.E. Ljance, Non-selfadjoint Differential Operators of Second Order on the Semiaxis. Supplement I to the book [17].

[19] S.A. Orlov, Nested Matrix Discs that Depend Analytically on a Parameter, and Theorems on the Invariance of the Ranks of the Radii of the Limit Matrix Discs. Izv. Akad. Nauk SSSR 40 (1976), No. 3, 593-644. (Russian) (Engl. transl.: Math. USSR-Izv. 10 (1976), 565-613).

[20] A.M. Kholkin, Description of Selfadjoint Extensions of Differential Operators of Arbitrary Order on an Infinite Interval in the Absolutely Indeterminate Case. Teor. Funkts., Funkts. Anal. Prilozhen. 1985, No. 44, 112-122. (Russian) (Engl. transl.: J. Sov. Math. 48 (1990), No. 3, 337-345).

[21] J.F. Brasche, M. Malamud, and H. Neidhardt, Weyl Function and Spectral Properties of Selfadjoint Extensions. - Integral Equations Operator 43 (1990), No. 3, 264-289.

[22] V.A. Derkach, S. Hassi, M.M. Malamud, and H.S.V. Snoo, Weyl Functions and Intermediate Extensions of Symmetric Operators. - Dopov. Nats. Akad. Nauk Ukr. (Mat. Prirodozn. Tekh. Nauki) (2001), No. 10, 33-39.

[23] V.I. Khrabustovskiy, On the Characteristic Matrix of Weyl-Titchmarch Type for Differential-Operator Equations with the Spectral Parameter Entering Linearly or by Nevanlinna Type. - Mat. Fiz. Anal. Geom. 10 (2003), No. 2, 205-227. (Russian)

[24] M. Lesch and M.M. Malamud, On the Deficiency Indices and Selfadjointness of Symmetric Hamiltonian Systems. — J. Diff. Equations 10 (2003), 556-615. 